\title{
Gastrointestinal endoscopy in patients with obstructive sleep apnea syndrome
}

\author{
Felix del Campo $\cdot$ Carlos Zamarrón
}

Received: 31 July 2011 /Revised: 10 August 2011 / Accepted: 12 August 2011 / Published online: 27 August 2011

(C) Springer-Verlag 2011

Patients with obstructive sleep apnea syndrome (OSAS) may be exquisitely sensitive to central nervous system depressant drugs showing potential for upper airway obstruction or apnea with even minimal doses of these drugs $[1,2]$. The anesthetic complications in OSAS patients may be due to multiple factors including collapse of the upper airway, type of surgery and anesthesia, and other associated conditions such as obesity. Since gastrointestinal endoscopy is a medical procedure requiring an anesthetic or analgesic, complications may be expected to occur more frequently in patients with OSAS.

For years, surgical risks for OSAS patients have been underestimated [3]. More recently, specific recommendations for the surgical care of patients with OSAS have been introduced in the guidelines of different anesthetic societies [4], in specific statements of management of patients with OSAS [5, 6], and more recently in the standards of sedation of patients who undergo gastrointestinal endoscopy [7]. In this regard, the Clinical Practice Review Committee of the American Academy of Sleep Medicine [8] has recommended that patients with a high index of suspicion for OSAS who are undergoing surgical procedures have (1) airway monitoring throughout the

F. del Campo $(\square)$

Division of Respiratory Medicine,

Hospital Universitario Río Hortega,

Dulzaina 2,

471012 Valladolid, Spain

e-mail: fsas@telefonica.net

C. Zamarrón

Division of Respiratory Medicine,

Hospital Clínico Universitario,

Santiago de Compostela, Spain

e-mail: Carlos.Zamarron.Sanz@sergas.es surgical period, (2) judicious use of appropriate anesthetic medication, and (3) monitoring throughout the process, especially during the postoperative period. Subsequently, the American Society of Anesthesiology [4] published a practical guide to the anesthetic care of OSAS patients. The guide was a consensus document based on existing literature and expert opinion, but as with other guides, implementation in clinical practice has been slow.

Gastrointestinal endoscopy is a safe procedure with a low event rate of serious cardiopulmonary complications. To make gastrointestinal endoscopic procedures better tolerated by the patient, utilization of light-to-moderate sedation is considered standard, and its use is routine. Most sedating medications used in anesthesia reduce the activity of airway muscles, favoring airway collapse. In OSAS patients, this phenomenon is aggravated by decreased ventilatory responses to hypoxia and hypercapnia and inhibited arousal responses to protect against apnea. Furthermore, obesity must be taken into account, in that obesity itself increases operative risk and is highly associated with OSAS. Furthermore, some of the drugs used in sedation must be adjusted to ideal weight.

Although the problem frequently arises in clinical practice, only a few studies, mainly retrospective, have evaluated the cardiovascular complications in patients with OSAS who were subjected to a digestive endoscopic procedure under light-to-moderate sedation [9-14]. In some studies, a clinical questionnaire was used before endoscopy to stratify patients into categories of high or low probability for OSAS. The Berlin questionnaire [15] was used by Khiani [9] and Mador [12]. Assessing the incidence of desaturations $(<92 \%)$ during endoscopy, Khiani found no differences between groups at low and high risk for OSAS. The Mador study [12], published in this issue of Sleep and Breathing, showed that patients with high probability of OSAS who are subjected to gastrointestinal endoscopic 
procedures have no greater number of complications, both major and minor compared to patients with low probability of OSAS. These findings do not support the research by Cote [13] that demonstrated more oxygen desaturations and ventilatory problems in patients with suspected OSAS using a clinical questionnaire (STOPBANG), but in this study, the patients had advanced endoscopic procedures.

With respect to studies involving a polysomnographic diagnosis, Mador [10] did not find a higher number of complications in patients with OSAS, regardless of the cutoff for diagnosis of OSAS or its severity. However, polysomnography was performed before endoscopy in only $63.5 \%$ of cases and preceded the procedure by a median of 1.84 years. In a retrospective study, Adler [11] claimed the safe use of propofol in endoscopic procedures finding no significant differences in the number of complications in patients with OSAS and patients without OSAS. However, a limitation of this study was that it did not account for patients treated with CPAP. Gill [14], using a case-control design in a veteran population, did not find differences in the number of complications from endoscopic procedures.

Another aspect that should be considered is the administration of oxygen therapy in routine endoscopy. One of the most frequent cardiorespiratory complications in patients who undergo endoscopy with or without sedation is transient hypoxemia which can range in frequency between $10 \%$ and $70 \%$, depending on the definition of hypoxemia, the underlying disease, previous baseline saturation, type of endoscopy and sedation used. The mechanisms of hypoxemia in this situation are multifactorial, but hypoventilation plays a key role. The use of oxygen is common in most endoscopy units. Its use can prevent the occurrence of hypoxemia, although its routine use is controversial especially in patients with COPD. Furthermore, it is not clearly established what constitutes an adequate oxygen flow and how protocols for the administration of oxygen should change in the presence of comorbidities such as obesity. Mador and colleagues [10] add to our understanding with their study demonstrating that the increased oxygen flow of $4 \mathrm{~L} / \mathrm{min}$ in obese patients reduces the number of desaturations.

In conclusion, evidence to date suggests that the presence of OSAS is not a contraindication for the use of sedation in gastrointestinal endoscopic procedures when properly monitored, regardless of the type of sedation and particular endoscopic procedure performed.

\section{References}

1. Adesanya AO, Lee W, Greilich NB, Joshi GP (2010) Perioperative management of obstructive sleep apnea. Chest 138:1489-1498

2. Mickelson SA (2007) Preoperative and postoperative management of obstructive sleep apnea patients. Otolaryngol Clin North Am 40:877-889

3. del Campo F, de Frutos J, Sánchez A (2008) Acute respiratory failure immediately following surgery for morbid obesity. Arch Bronconeumol 44:449-450

4. Gross JB, Bachenberg KL, Benumof JL, Caplan RA, Connis RT (2006) Practice guidelines for perioperative management of patients with obstructive sleep apnea: a report by the American Society of Anesthesiologists Task Force on Perioperative Management of patients with obstructive sleep apnea. Anesthesiology 104:1081-1093

5. Fleetham J, Ayas N, Bradley D, Fitzpatrick M, Oliver T, Morrison D, Ryan F, Series F, Skomro R, Tsai W (2006) Canadian Thoracic Society guidelines: diagnosis and treatment of sleep disordered breathing in adults. Can Respir J 13:387-392

6. Lloberes P, Durán-Cantolla J, Martínez-García MA, Marín JM, Ferrer A, Corral J, Masa JF, Parra O, Alonso-Álvarez ML, Terán-Santos J (2011) Diagnosis and treatment of sleep apnea-hypopnea syndrome. Arch Bronconeumol 47:143-156

7. Thomson A, Andrew G, Jones DB (2010) Optimal sedation for gastrointestinal endoscopy: review and recommendations. J Gastroenterol Hepatol 25:469-478

8. Meoli AL, Rosen CL, Kristo D, Kohrman M, Gooneratne N, Aguillard RN, Fayle R, Troell R, Kramer R, Casey KR, Coleman J Jr (2003) Upper airway management of the adult patient with obstructive sleep apnea in the preoperative period-avoiding complications. Sleep 26:1060-1065

9. Khiani VS, Salah W, Maimone S, Cummings L, Chak A (2009) Sedation during endoscopy for patients at risk of obstructive sleep apnea. Gastrointest Endosc 70:1116-1120

10. Mador MJ, Abo Khamis M, Nag N, Mreyoud A, Jallu S, Mehboob S (2010) Does sleep apnea increase the risk of cardiorespiratory complications during endoscopy procedures. Sleep Breath. doi:10.1007/s11325-010-0346-3

11. Adler DG, Kawa C, Hilden K, Fang J (2011) Nurse-administered propofol sedation is safe for patients with obstructive sleep apnea undergoing routine endoscopy: a pilot study. Dig Dis Sci. doi:10.1007/s10620-011-1645

12. Mador MJ, Nadler J, Mreyoud A, Khadka G, Gottumukkala VA, AboKhamis M, Mehboob S (2011) Do patients at risk of sleep apnea have an increased risk of cardio-respiratory complications during endoscopy procedures. Sleep Breath. doi:10.1007/s11325-011-0546-5

13. Cote GA, Hovis ChE, Hovis RM, Waldbaum L, Early DS, Edmundowicz SA, Azar RR, Mullady DK, Jonnalagadda SS (2010) A screening instrument for sleep apnea predicts airway maneuvers in patients undergoing advanced endoscopic procedures. Clin Gastroenterol Hepatol 8:660-665

14. Gill J, Vidyarthi G, Kulkarni P, Anderson W, Boyd W (2011) Safety of conscious sedation in patients with sleep apnea in a veteran population. South Med J 104:185-188

15. Chung F, Ward B, Ho J, Yuan H, Kayumov L, Shapiro C (2007) Preoperative identification of sleep apnea risk in elective surgical patients, using the Berlin questionnaire. J Clin Anesth 19:130-134 